Nota Bene: This manuscript has been submitted as a proceeding article for the European Navigation Conference 2020. Please note that manuscript has yet to be formally accepted for publication. Subsequent versions of this manuscript may have slightly different content. If accepted, the final version of this manuscript will be available via the Peer-reviewed Publication DOI link on the right-hand side of the EarthArXiv webpage. Please feel free to contact any of the authors; we welcome feedback.

\title{
A prototype for a Multi-GNSS orbit combination
}

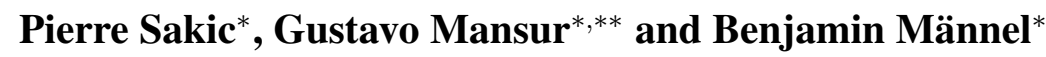

${ }^{*}$ GFZ German Research Centre for Geosciences, Helmholtz-Zentrum Potsdam, Potsdam, Germany

${ }^{* *}$ Technische Universität Berlin, Institute for Geodesy and Geoinformation Technology,

Berlin, Germany

email: pierre.sakic@gfz-potsdam.de

\begin{abstract}
Since 1994, the International GNSS Service (IGS) provides a combination of orbit and clock offset products from its different Analysis Centers. These products are used as input by many software for countless scientific and/or operational applications. They can also be used as independent reference for benchmark experiments. Nevertheless, those products include GPS/GLONASS-only data, despite the fact that nowadays several GNSS constellations are available. We performed modifications on the existing IGS combination software and made it compatible with multi-GNSS products. We present here the results of a combination of five Multi-GNSS Experiment Analysis Centers (ACs) for five constellations (GPS + GLONASS + Galileo + Beidou + QZSS) from GPS week 1800 to 2000 (July 2014 to May 2018). The RMS of combined orbit w.r.t. input ACs ones are $\sim 30 \pm 15 \mathrm{~mm}$ for recent weeks of the test period.
\end{abstract}

\section{Introduction}

The International GNSS Service (IGS) is a worldwide scientific organization gathering since the early 1990s numerous Geosciences institutes, universities, along with mapping and space agencies [16]. Some IGS members host so-called Analysis Centers (ACs), having the operational task to provide to the public on a continuous basis high accuracy products, i.e. station positions, satellite orbits \& clocks offsets, and Earth orientation parameters (EOPs) for instance.

The IGS Analysis Center Coordinator (ACC) uses all individual AC orbit products as input, to determine an combined orbit solution provided to the community. This combination consists 
basically of an aligned and weighted mean of all individual AC products. It will be called hereafter the legacy combination [18].

Historically, the IGS mainly focused its studies on precise orbit determination of the American GPS and the Russian GLONASS constellations ${ }^{1}$, the only two GNSS available at the Service early phase. Nevertheless, a new era in satellite navigation began in the late 2000s, when the first satellites of the new European constellation Galileo and the Chinese BeiDou have been launched. To accompany this transition to a multi-constellation environment, the IGS started in 2012 the so-called MGEX, standing for Multi-GNSS EXperiment [25], and continuing nowadays as a pilot project for Multi-GNSS Extension. The MGEX aims to enhance the global tracking network with stations able to record the maximum of new signals associated with the new systems or satellite generations, along with the enhancement of the different AC processing software. More and more new MGEX ACs (including some established IGS members) start to provide multi-GNSS products, i.e. containing namely Galileo, BeiDou, and QZSS ${ }^{2}$.

Nowadays, the multi-GNSS environment can be considered as enabled since Galileo, BeiDou and QZSS have been officially declared in their operational phases by their respective operating agencies $[6,3,15]$. Nevertheless, no official IGS combination including the new GNSS is provided so far, despite the fact that some preliminary studies have been performed by [?]. This constitutes an important limitation for the exploitation of the new GNSS for the scientific and engineering communities which usually use IGS products for its applications, in geophysics, oceanography, surveying, etc..

This article exposes the results for a preliminary multi-GNSS orbit combination based on an extension of the legacy combination software. We present hereafter the modifications performed on the software, and the different multi-GNSS AC products used for a test period of 200 weeks between the GPS weeks 1800 and 2000 (July 2014 to May 2018). The orbit combination results are compared with each individual AC, for all satellites and for each separated constellation.

\section{Modifications to the Combination Software}

The combination strategy follows the method developed by [34] and described in more details by [19]. The general workflow and can be summarized as follows:

1. A preliminary alignment step, where each AC orbit solution is transferred to a common reference. Historically, for the legacy combination, this alignment was performed with a rotation of the residual values between the Earth Orientation Parameters (EOP) determined by each AC and the ones provided by IERS Bulletin B [19]. Since 2000, this alignment is done directly based on the IGS realization of the International Terrestrial Reference Frame using the transformation parameters provided by the IGN [31]. Those

\footnotetext{
${ }^{1}$ GLONASS observations started to be considered in the frame of the IGEX campaign in 1998 .

${ }^{2}$ Stricto sensu, QZSS is a Regional Navigation Satellite System, since it covers only the Asia-Oceania regions, with a focus on Japan.
} 
two strategies are equivalent: the underlying idea is to remove potential misalignments at the ACs level. Nevertheless, the latter can be considered as better, since the alignment is done with respect to the IGS reference frame. Unfortunately, this second approach cannot be used, since the transformation parameter are not available yet. Thus, for the current work, this step is done on the EOP approach.

2. A first equally weighted orbit mean is estimated.

3. An Helmert transformation is estimated between each AC solution and the step 2 mean.

4. Weights for each AC are computed based on the formula:

$$
W_{c e n t}=\left[\frac{\left(3 \cdot \sum_{s a t}^{S_{c e n t}} E_{c e n t}^{s a t}\right)-7}{\sum_{\text {sat }}^{S_{c e n t}} \sum_{i}^{\text {sat }} \sum_{i}^{\text {cent }}\left|P_{c e n t}^{\prime s a t}-\bar{P}^{s a t}\right|_{i}}\right]^{2}
$$

where $S_{\text {cent }}$ is the number of satellites per AC, $E_{\text {cent }}^{\text {sat }}$ is the number of positions per AC per satellite. The position of the transformed orbits and the mean orbit is represented by $P_{\text {cent }}^{\prime \text { sat }}$ (step 3) and $\bar{P}^{\text {sat }}$ (step 2), respectively.

5. The weighted mean is estimated based on:

$$
\bar{P}_{\text {comb }}^{\text {sat }}=\frac{\sum_{\text {cent }}^{N_{\text {cent }}^{\text {sat }}} W_{\text {cent }} P_{\text {cent }}^{\prime \prime} \text { sat }}{\sum_{\text {cent }}^{N_{\text {cent }}^{\text {sat }}} W_{\text {cent }}}
$$

Where $N_{\text {cent }}^{\text {sat }}$ is the number of Centers submitting a solution for that satellite, and $P_{\text {cent }}^{\prime \prime}$ sat is an AC solution realigned on the step 2 mean but using a satellite-weighted Helmert transformation (see [19] for details).

6. Satellite outliers are determined: if the RMS of a satellite computed by an AC exceeds three times the overall satellite RMS mean used as a threshold, then this AC's satellite is excluded for the next iteration. Note that an exclusion does not mean that the satellite will be completely eliminated. It means only that this satellite will not participate in the determination of Helmert transformation parameters and weights, but a combined orbit for an excluded satellite is still provided using the $\mathrm{AC}$ weight computed based on the valid satellites. Note also that the software can manage satellite provided by only one AC: if a satellite is included in only one input, it will be included in the combined solution as it is (but still corrected of the pole alignment, step 1).

7. While there is still a satellite exclusion, the combination restarts at step 2 . 
This method can be considered as reliable, since only few modifications have been performed on it since the mid-1990, and it is still this software which is used on a continuous basis by the ACC.

We started from a modified version of the original ACC combination software designed for a GPS and Galileo orbit combination within the GGSP consortium (Galileo Geodetic Service Provider) [7, 32], and performed the necessary modifications to make it capable of handling the new constellations. The improvements carried out are:

- A proper handling of the new Galileo, BeiDou and QZSS constellations for the Pole Alignment, Satellite \& AC weight determination and the final statistics computations.

- The handling of the SP3- $d$ format, i.e. the most suited one for a multi-GNSS environment, since this version can support an unlimited number of satellites [12]. The previously implemented SP3-c format could only handle 85 satellites, and this limit has been reached for the last weeks of the test period (GFZ products include 86 satellites starting from the day 1947-6, 2017/05/06).

Nevertheless, no modification on the algorithm itself has been made. Thus, no specific weighting is applied for each constellation. Then, internally, all satellites are assumed as belonging to one and the same "big" constellation. We used this strategy instead of a constellationindependent combination, to maintain consistency in the AC weighting for the different constellations, in the continuity of the scheme designed for the GGSP [7].

\section{Products used}

We focused our multi-GNSS combination test study for a period of 200 weeks, from GPS week 1800 to week 2000 (from 2014/07/06 to 2018/05/06). During this period, Multi-GNSS products were provided by the following Analysis Centers:

- Center for Orbit Determination in Europe (CODE), Bern [30, 29];

- Centre National d'Études Spatiales (CNES), Groupe de Recherche de Géodésie Spatiale (GRGS), Toulouse [21, 17];

- Deutsches GeoForschungsZentrum (GFZ), Potsdam [8, 5]

- Japan Aerospace Exploration Agency (JAXA), Tōkyō [36];

- Shanghai Astronomical Observatory (SHAO), Shanghai [2]

- Technische Universität München (TUM), Munich [33]; 
- Wuhan University (WU), Wuhan [11].

TUM products containing only orbits for Galileo, QZSS and BeiDou were not used in this study. Indeed, for historical reasons, the combination software necessarily requires GPS data to work properly, and adequate modifications would have been beyond the scope of the enhancements we wished. SHAO products covered a too short time span of only few weeks at the end of our study period (from week 1990 on CDDIS server [26]). Therefore, we did not use them. Lastly, we precise that the ESOC, Darmstadt also generates multi-GNSS products [24], but they are not submitted to the MGEX project, thus they were not used in our study.

Hereafter, we use the RINEX format conventional abbreviations for each GNSS: G for GPS, $\mathrm{R}$ for GLONASS, E for Galileo, $\mathrm{C}$ for BeiDou, $\mathrm{J}$ for QZSS. The different AC products per constellation processed during the test period are represented in Figure 1(a), and the number of satellites used in the combination in Figure 1(b). We note that during the test period, GRGS and JAXA started to provide their products (from week 1826 and 1945 respectively on CDDIS server, GFZ started to process GLONASS and Galileo (from day 1820-4, 2014/11/27) along with QZSS (from day 1846-3, 2015/05/27), and Wuhan University started to process QZSS from day 1826-3, 2015/01/07.
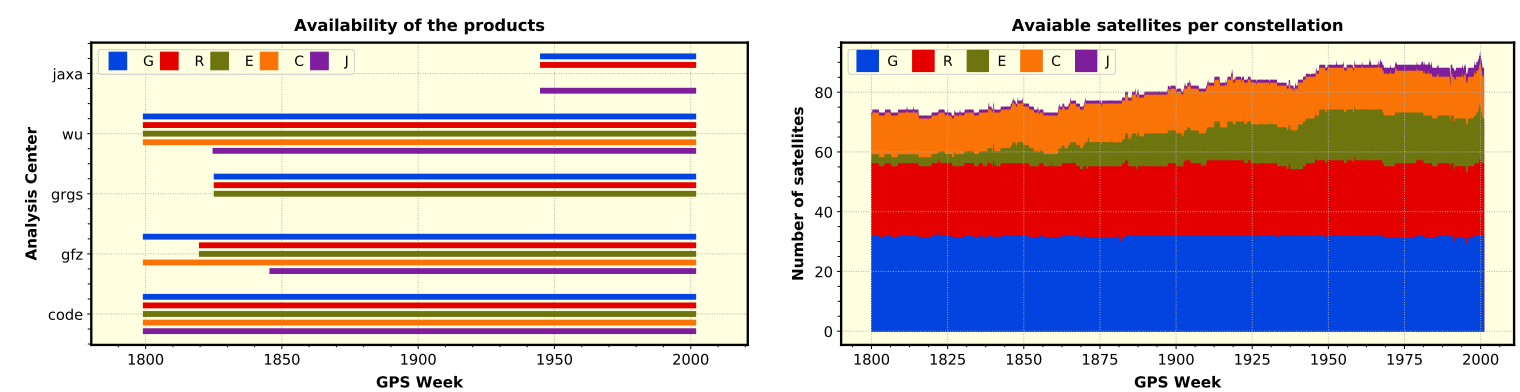

(a) Timeline of the data provided by the different Anal- (b) Representation of the total number of satellites ysis Centers within the test period.

available for the combination per constellation.

Figure 1: Timelines of the available products (system and satellites) during the test period

As mentioned above, the first step of the combination is an alignment to a common reference frame. This is done using the EOP approach described in section 1. Nevertheless, some ACs do not provide EOP data in the dedicated erp format (namely TUM and GRGS, the latter providing their EOP estimation in the SINEX file). Thus, no preliminary alignment is performed and they are assumed to be correctly pre-aligned to the common reference, This step aims to reduce at the maximum the standard deviation of the first mean combination.

\section{Orbit combination results}

The RMS differences between the combined solution and the individual ACs solution for all satellites are computed using the formulas described in [19]. They are represented in Figure 

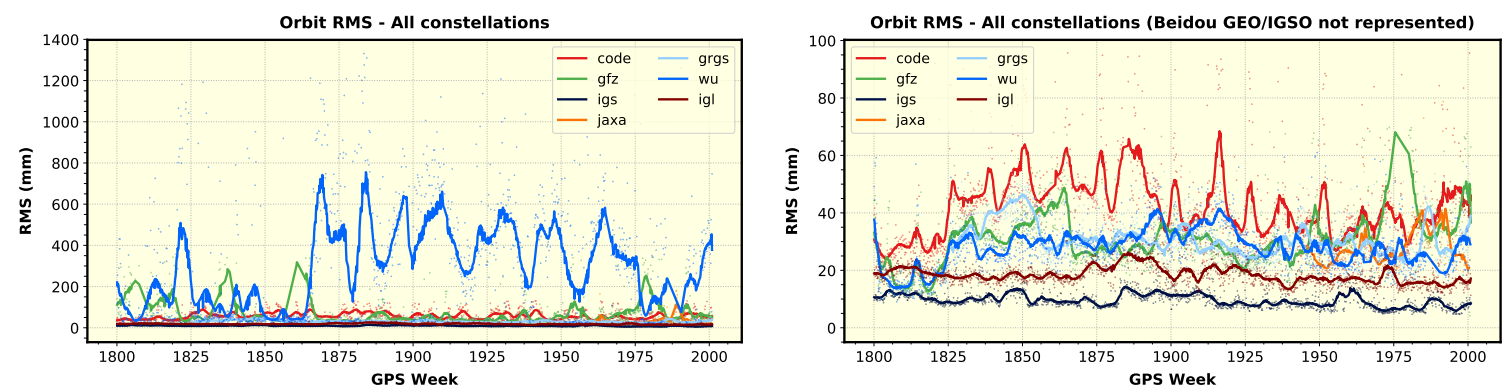

(a) all constellations, with BeiDou GEO/IGSO satellites

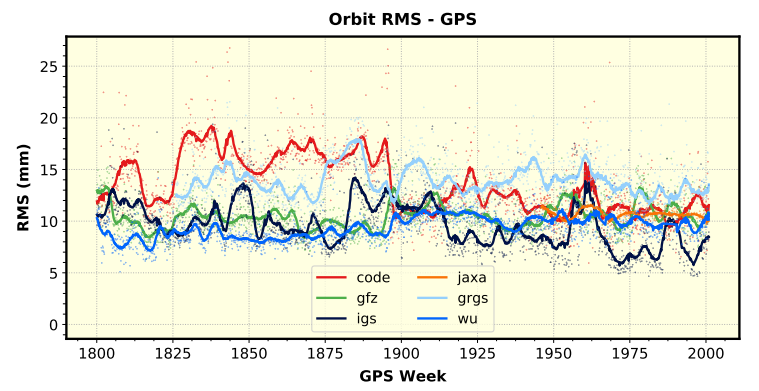

(b) all constellations, without BeiDou GEO/IGSO satellites

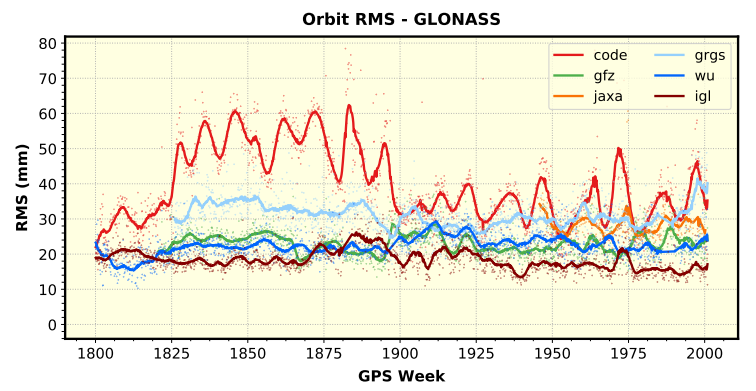

(c) GPS

(d) GLONASS
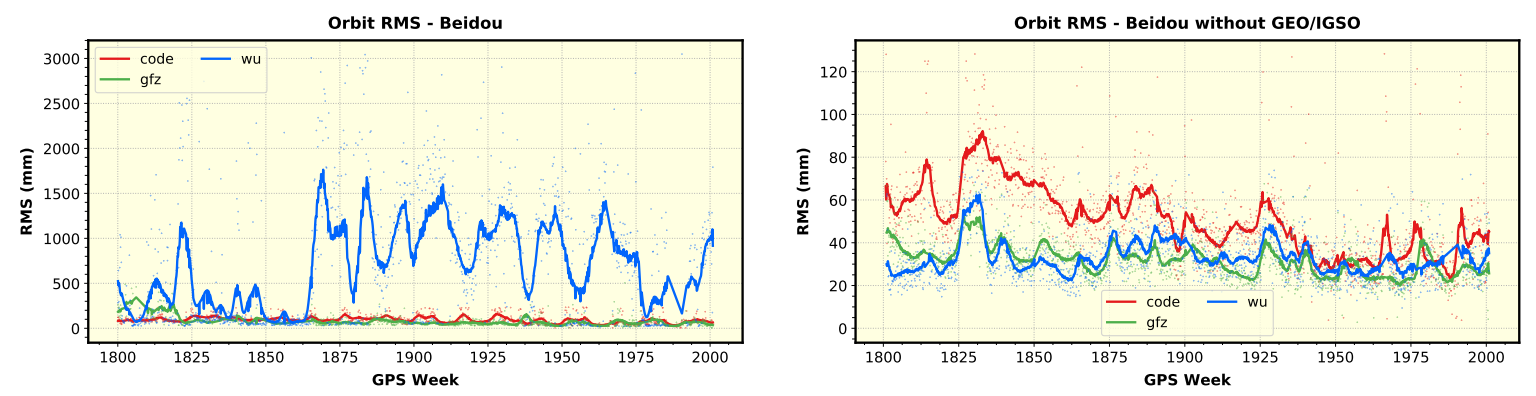

(e) BeiDou, with BeiDou GEO/IGSO satellites

(f) BeiDou, without BeiDou GEO/IGSO satellites

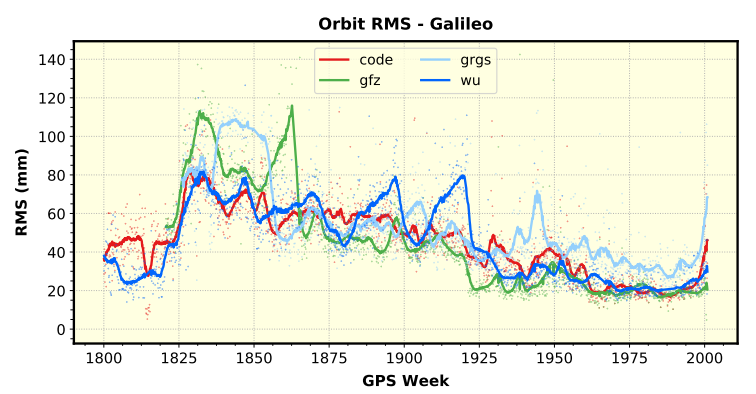

(g) Galileo

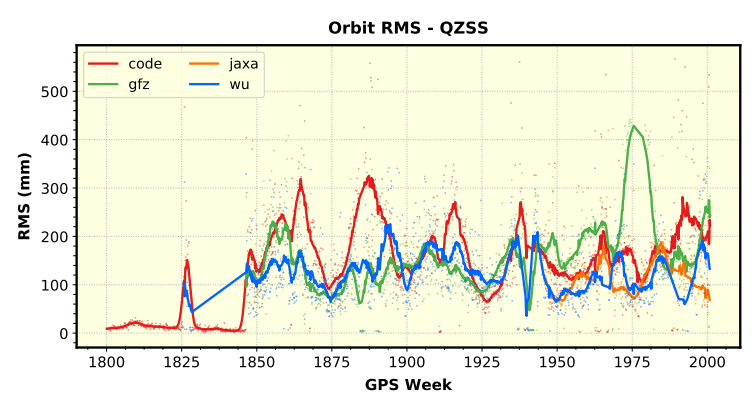

(h) QZSS

Figure 2: 3D-RMS difference of each individual AC orbit products with respect to the combined orbit solution for the different constellations (different y-axis scales)

2(a). We use a similar representation as the ones usually provided by the ACC: the daily RMS are represented by dots, and a smoothing curve based on a Gaussian filter (with a 7-day window 
in our case) is used to represent the long-term tendency. We remark that important differences are dominated by WU solution, due to the BeiDou geostationary satellites (hereafter designated as GEO) which degrade their general orbit RMS. The GEO orbit determination is known to be more difficult, since the satellite position variations with respect to the ground tracking network is small, especially on the along-track component [13]. The attitude law for BeiDou-2 GEO (orbit-normal) is also different from the regular yaw-steering law normally used for GNSS [20] and require a proper implementation in the AC processing software. If we exclude the BeiDou GEO and IGSO (inclined geosynchronous satellites) from the RMS difference computation (Figure 2(b)), the RMS difference for all ACs is around $30 \pm 15 \mathrm{~mm}$. We decided to exclude IGSO too in Figure 2(b), even if their consistency is one order of magnitude better than the geostationary ones [35], in order to have a MEO-only visualization more consistent with the other constellations.

Figures 2(a) to 2(h) represent the RMS differences for each individual constellation. For GPS, differences of individual $\mathrm{AC}$ with respect to the combination is between $8 \mathrm{~mm}$ and $18 \mathrm{~mm}$ with an average value around $10 \mathrm{~mm}$ for the second part of the test period. The differences with the IGS legacy combination (in dark blue for GPS, in dark red for GLONASS) are also shown, with a RMS value below the centimeter level for the second part of the test period for GPS and around $20 \mathrm{~mm}$ for GLONASS. RMS differences with respect to the individual AC solutions for GPS is at the centimeter level, which is consistent with the average difference obtained for the legacy combination [14] (between $9 \mathrm{~mm}$ and $20 \mathrm{~mm}$ for 10 ACs when these lines are written i.e. for a representative period between weeks 1990 and 2055) or for the repro2 campaign combination [9] (between $8 \mathrm{~mm}$ and $13 \mathrm{~mm}$ for 10 ACs for a representative period between weeks 1250 and 1831). For GLONASS (Figure 2(d)), the differences are between $20 \mathrm{~mm}$ and $60 \mathrm{~mm}$ with an average around $25 \mathrm{~mm}$ for the second part of the test period. We remark that when GRGS starts to provide GLONASS (on week 1826) the CODE solution RMS increase brutally, meaning that a consistent solution between the other ACs is then reinforced with the new inputs. This behavior is corrected around week 1900, when CODE implemented new attitude models in their processing according to IGS technical report [38]. Furthermore, this improvement is also visible for GPS (Figure 2(c)).

Regarding the new constellations, a noticeable improvement of Galileo orbit quality can be remarked (Figure $2(\mathrm{~g})$ ), since the difference of individual $\mathrm{AC}$ with respect to the combination decreased within a processed period of 200 weeks from $100 \mathrm{~mm}$ to $20 \mathrm{~mm}$. The visible increase of the RMS around weeks 1944 and 2000, especially for GRGS might correspond to the new satellite activation of launches L8 and L9 respectively. The IOV (In Orbit Validation) Satellite Metadata release in December 2016 can explain the general RMS decrease visible around week 1925. The similar release for the FOC (Full Operational Capability) satellites in October 2017 can explain the stabilization of the RMS around $20 \mathrm{~mm}$ after week 1971. For BeiDou (Figure 2(e)), a similar tendency exists as for the plot showing all the constellations: the geostationary satellites lead the WU solution differences at the metric level. But when we represent only the Medium Earth Orbit (MEO) satellites (Figure 2(f)), an RMS difference of $\sim 30 \mathrm{~mm}$ is achieved (up to $\sim 90 \mathrm{~mm}$ for CODE but decreasing over time). For QZSS, (Figure 2(h)), the 
RMS difference, although stable, are significantly higher, centered around $\sim 150 \mathrm{~mm}$, but with variation from $\sim 80 \mathrm{~mm}$ up to $\sim 420 \mathrm{~mm}$.

\section{SLR residuals}

We used Satellite Laser Ranging (SLR) observations to perform an independent quality assessment of the combination. All active Beidou, Galileo, GLONASS, and QZSS satellites are suitable for such analysis since they are equipped with Laser Retroreflector Arrays (LRA, [4]). We used as input the normal points provided by the International Laser Ranging Service [28]. The processing is performed with GFZ's EPOS8 software [37], designed for GNSS precise orbit determination and SLR residual estimation. SLR station coordinates are fixed to SLRF2008 [27] before the official IGS switch to the ITRF2014 [1] on January 21, 2016 and SLRF2014 after [22]. Station positions are corrected of ocean tidal loading using the FES2004 model [23]. Residuals over $0.5 \mathrm{~m}$ are considered as outliers and removed. Daily averaged residuals are shown in Figure 3.

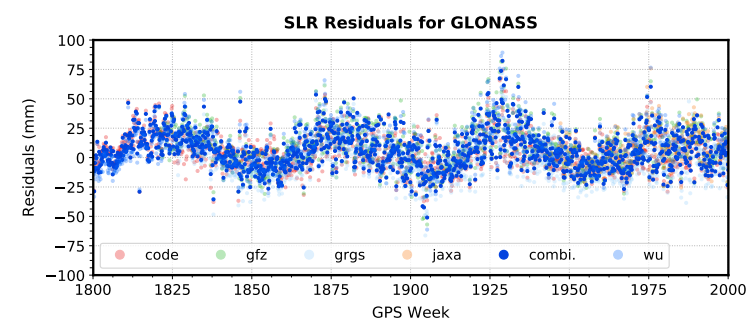

(a) GLONASS

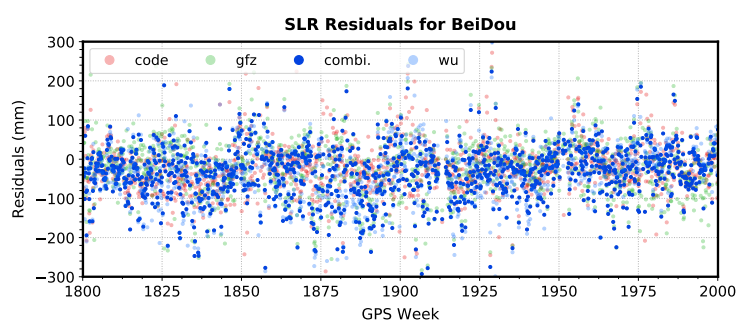

(c) BeiDou

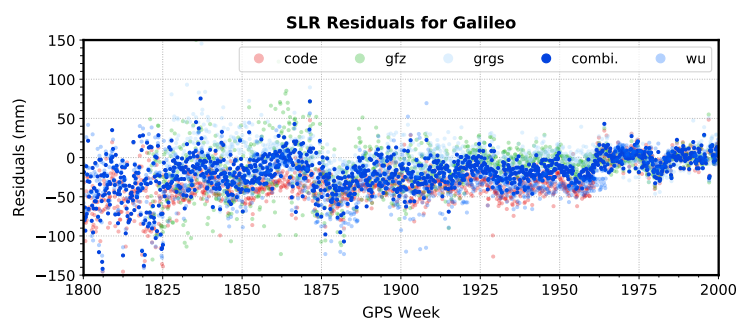

(b) Galileo

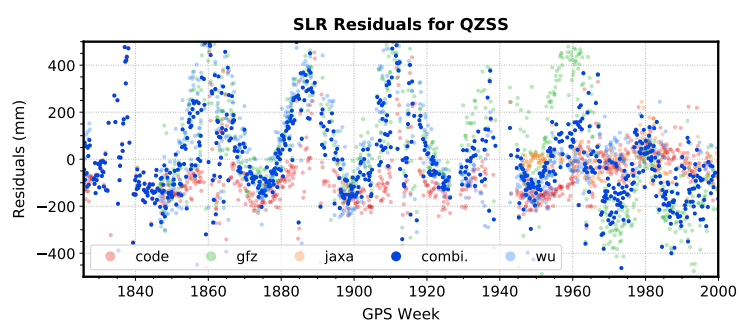

(d) QZSS

Figure 3: Average SLR Residuals per constellation for each AC solution and the combination (different y-axis scales).

The combination, in blue, shows a smaller dispersion than the individual input solution, especially for Galileo. The effect of the FOC Metadata release after week 1971 is clearly visible: a bias is corrected and the SLR residual are then centered on zero. 


\section{Discussion and perspectives}

We modified the existing IGS orbit combination software to make it capable of handling the new GNSS constellations, namely Galileo, BeiDou and QZSS. The first results are encouraging. This experimental Multi-GNSS combination can be validated while comparing with the legacy combination: the consistency between both combinations for GPS is below the centimeter level.

Nevertheless, some important weaknesses have been raised, especially regarding the exclusion depending on AC/constellation activation. First, since all satellites are considered as belonging to a unique and common constellation, there is no special weighting according to each navigation system. This new feature would be nevertheless highly valuable. Indeed, some constellations should be considered as more reliable since their orbits are more precisely determined, like GPS. Thus, they should get a higher weight in the combination process. The fact that the European GNSS Agency provides for Galileo metadata such as attitude laws and accurate satellite geometry [10] can also be a plus feature for this constellation. Moreover, there is no special handling for satellites with new kind of orbits, such as geostationary orbit and inclined geosynchronous orbits (some BeiDou satellites and QZSS) or even the two elliptical orbit Galileo satellites. Since it is more challenging to achieve a highly accurate orbit determination for those satellites because of different orbit parameterization and software capabilities, they penalize the weight of the AC which process them, even if the determined orbits for more well-modeled satellites (i.e. circular MEO such as GPS vehicles) are precise enough. Moreover, the necessity of a combined product for those "unconventional" satellites is maybe the most relevant, since the differences between individual solutions appear larger. Lastly, the alignment to a common reference, which is the prerequisite step before the weight determination, need Earth Orientation Parameters data. Unfortunately, some ACs does not provide such products. The preliminary alignment is then skipped for some ACs, which biases the combination. Then, we would like to encourage MGEX Analysis Centers to provide a full set of Multi-GNSS products, including especially the EOP.

Thus, and even if the current IGS software could actually handle the new GNSS constellation and achieve a relevant combination quite easily, it would be beneficial to start the development of a new algorithm. Several ideas for this new strategy are under study: we first developed a workaround method to align the different MGEX solution on a common pole reference [?]. We also started to develop an optimized weighting scheme, considering the different constellations.

\section{References}

[1] Z. Altamimi, P. Rebischung, L. Métivier, and X. Collilieux. ITRF2014: A new release of the International Terrestrial Reference Frame modeling nonlinear station motions. Journal of Geophysical Research: Solid Earth, 121(8):6109-6131, aug 2016.

[2] J. Chen, B. Wu, X. Hu, and H. Li. SHA: The GNSS Analysis Center at SHAO. In J. Sun, W. Jiao, $\mathrm{H}$. Wu, and C. Shi, editors, China Satellite Navigation Conference (CSNC) 2012 Proceedings, vol- 
ume 243 of Lecture Notes in Electrical Engineering, pages 213-221. Springer Berlin Heidelberg, Berlin, Heidelberg, 2012.

[3] CSNO. The BDS-3 Preliminary System Is Completed to Provide Global Services, 2018.

[4] S. Dell'Agnello, G. O. Delle Monache, D. G. Currie, R. Vittori, C. Cantone, M. Garattini, A. Boni, M. Martini, C. Lops, N. Intaglietta, R. Tauraso, D. A. Arnold, M. R. Pearlman, G. Bianco, S. Zerbini, M. Maiello, S. Berardi, L. Porcelli, C. O. Alley, J. F. McGarry, C. Sciarretta, V. Luceri, and T. W. Zagwodzki. Creation of the new industry-standard space test of laser retroreflectors for the GNSS and LAGEOS. Advances in Space Research, 47(5):822-842, 2011.

[5] Z. Deng, M. Fritsche, T. Nischan, and M. Bradke. Multi-GNSS ultra rapid orbit-, clock- and EOPproduct series, 2016.

[6] ESA. Galileo begins serving the Globe, 2016.

[7] G. Gendt, Z. Altamimi, R. Dach, W. Söhne, and T. Springer. GGSP: Realisation and maintenance of the Galileo Terrestrial Reference Frame. Advances in Space Research, 47(2):174-185, 2011.

[8] G. Gendt, G. Dick, and W. Söhne. GFZ Analysis Center of IGS-Annual Report. IGS 1996 Annual Report, pages 169-181, 1998.

[9] J. Griffiths. Combined orbits and clocks from IGS second reprocessing. Journal of Geodesy, 93(2):177-195, feb 2019.

[10] GSA. Galileo Satellite Metadata, 2017.

[11] J. Guo, X. Xu, Q. Zhao, and J. Liu. Precise orbit determination for quad-constellation satellites at Wuhan University: strategy, result validation, and comparison. Journal of Geodesy, 90(2):143-159, 2016.

[12] S. Hilla. The Extended Standard Product 3 Orbit Format (SP3-d). Technical report, National Geodetic Survey, Silver Spring, 2016.

[13] U. Hugentobler and O. Montenbruck. Satellite Orbits and Attitude. In Springer Handbook of Global Navigation Satellite Systems, pages 59-90. Springer International Publishing, Cham, 2017.

[14] IGS-ACC. Figures and Statistics from the IGS Final GPS Orbit Combination, 2012.

[15] JAXA. Start of QZSS Services, 2018.

[16] G. Johnston, A. Riddell, and G. Hausler. The International GNSS Service. In Springer Handbook of Global Navigation Satellite Systems, pages 967-982. Springer International Publishing, Cham, 2017.

[17] G. Katsigianni, S. Loyer, F. Perosanz, F. Mercier, R. Zajdel, and K. Sośnica. Improving Galileo orbit determination using zero-difference ambiguity fixing in a Multi-GNSS processing. Advances in Space Research, 63(9):2952-2963, may 2019.

[18] J. Kouba. A Guide to using international GNSS Service (IGS) Products. Technical report, 2015.

[19] J. Kouba, Y. Mireault, and F. Lahaye. IGS orbit/clock combination and evaluation, Appendix 1 of the analysis coordinator report, international GPS Service For Geodynamics 1994 Annual Report. Jet Propulsion Laboratory publication, pages 18-95, 1994.

[20] X. Li, Y. Yuan, Y. Zhu, J. Huang, J. Wu, Y. Xiong, X. Zhang, and X. Li. Precise orbit determination for BDS3 experimental satellites using iGMAS and MGEX tracking networks. Journal of Geodesy, 93(1):103-117, 2019.

[21] S. Loyer, F. Perosanz, L. Versini, G. Katsigianni, F. Mercier, and A. Mezerette. CNES/CLS IGS Analysis center: recent activities. In IGS Workshop 2018, Wuhan, 2018.

[22] V. Luceri, E. C. Pavlis, B. Pace, D. König, M. Kuzmicz-Cieslak, and G. Bianco. Overview of the ILRS Contribution to the Development of ITRF2013. In International Association of Geodesy Symposia, pages 101-108. 2015. 
[23] F. Lyard, F. Lefevre, T. Letellier, and O. Francis. Modelling the global ocean tides: Modern insights from FES2004. Ocean Dynamics, 56(5-6):394-415, 2006.

[24] V. Mayer, T. Springer, E. Schönemann, and W. Enderle. ESA Multi-GNSS Products. In EGU General Assembly 2019, volume 21, 2019.

[25] O. Montenbruck, P. Steigenberger, L. Prange, Z. Deng, Q. Zhao, F. Perosanz, I. Romero, C. Noll, A. Stürze, G. Weber, R. Schmid, K. MacLeod, and S. Schaer. The Multi-GNSS Experiment (MGEX) of the International GNSS Service (IGS) - Achievements, prospects and challenges. $A d$ vances in Space Research, 59(7):1671-1697, 2017.

[26] C. E. Noll. The crustal dynamics data information system: A resource to support scientific analysis using space geodesy. Advances in Space Research, 45(12):1421-1440, jun 2010.

[27] E. C. Pavlis, V. Luceri, C. Sciarretta, and R. Kelm. The ILRS Reanalysis 1983-2009 Contributed To ITRF2008. In AGU Fall Meeting Abstracts, 2009.

[28] M. Pearlman, J. Degnan, and J. Bosworth. The International Laser Ranging Service. Advances in Space Research, 30(2):135-143, jul 2002.

[29] L. Prange, D. Arnold, R. Dach, S. Schaer, D. Sidorov, P. Stebler, A. Villiger, and A. Jäggi. CODE product series for the IGS-MGEX project. 2018.

[30] L. Prange, E. Orliac, R. Dach, D. Arnold, G. Beutler, S. Schaer, and A. Jäggi. CODE's fivesystem orbit and clock solution-the challenges of multi-GNSS data analysis. Journal of Geodesy, 91(4):345-360, 2017.

[31] P. Rebischung, Z. Altamimi, J. Ray, and B. Garayt. The IGS contribution to ITRF2014. Journal of Geodesy, 90(7):611-630, 2016.

[32] P. Sakic, B. Männel, T. Nischan, and GGSP. Operational geodetic products determination and combination for Galileo. In EGU General Assembly 2018, volume 20, 2018.

[33] I. Selmke, B. Duan, and U. Hugentobler. Status of the TUM MGEX orbit and clock products. In IGS Workshop 2018, Wuhan, 2018.

[34] T. Springer and G. Beutler. Towards an official IGS orbit by combining the results of all IGS Processing Centers. In Proceedings of the 1993 IGS Workshop, held March, pages 24-26, 1993.

[35] P. Steigenberger, U. Hugentobler, A. Hauschild, and O. Montenbruck. Orbit and clock analysis of Compass GEO and IGSO satellites. Journal of Geodesy, 87(6):515-525, 2013.

[36] T. Takasu, M. Miyoshi, K. Kaori, and K. Satoshi. QZSS-1 Precise Orbit Determination by MADOCA. In International Symposium on GNSS 2015, Kyoto, 2015.

[37] M. Uhlemann, G. Gendt, M. Ramatschi, and Z. Deng. GFZ Global Multi-GNSS Network and Data Processing Results. In International Association of Geodesy Symposia, volume 12, pages 673-679. Springer, 2015.

[38] A. Villiger and R. Dach. IGS Technical Report 2016. Technical report, IGS Central Bureau and University of Bern, Bern, 2017. 